\title{
Determinantes naturales y antrópicos de la distribución, estructura y composición florística de los manglares de la Reserva Natural Sanguaré, Colombia
}

\author{
Juan David Mira, Ligia Estela Urrego \& Katherine Monsalve ${ }^{1}$ \\ Universidad Nacional de Colombia, Facultad de Ciencias Agrarias, Departamento de Ciencias Forestales, A.A. 568 \\ Medellín; judmirama@unal.edu.co, leurrego@unal.edu.co,kmonsalvec@unal.edu.co \\ * Correspondence
}

Recibido 08-XI-2018. Corregido 19-III-2019. Aceptado 04-VII-2019.

\begin{abstract}
Natural and anthrophic deteminants of distribution, structure and floristic composition of mangroves in the Natural Sanguaré Reserve, Colombia. Mangroves have different degrees of interaction between oceanographic, climatic, geomorphological and soil conditions. Those interactions have strong impacts on local salinity conditions and availability of water and nutrients at local level, and on mangrove structure and composition. Based on the analysis of aerial photographs, field sampling and statistical multivariate analysis, floristic composition and structure of the mangroves present in the Sanguare Natural Reserve were analyzed. Three types of forests were identified according to the physiographic classification of mangroves. A fridge mangrove dominated by Rhizophora mangle L. in a juvenile state, with low structural development, and two types of inland or basin mangroves dominated by Avicennia germinans (L.) L. The first of them, located behind the fridge mangrove, showed high salinity in the soil and in the interstitial water, predominance of sandy sediments, and low values of height and diameter at breast height (DBH) average of the trees. The second, with a low number of adult trees in all diameter categories, high basal area, low natural regeneration potential, and high concentrations of $\mathrm{Fe}$ and $\mathrm{Cu}$ in the soil, probably associated with contamination by domestic spills. The location of the first inner mangrove behind the fridge mangrove reveals the typical zonation of many mangroves in the world. The floristic composition, structure and distribution of the mangroves in the Sanguare Reserve are the result of the interaction of local environmental variables such as salinity and soil texture, as well as the anthropic intervention prior to the establishment of the reserve and the current human presence being very close to the mangroves.
\end{abstract}

Key words: mangrove; regeneration; contamination; natural variability; conservation.

Mira, J. D., Urrego, L. E. \& Monsalve, K. (2019). Determinantes naturales y antrópicos de la distribución, estructura y composición florística de los manglares de la Reserva Natural Sanguaré, Colombia. Revista de Biología Tropical, 67(4), 810-824.

La estructura y la composición florística de los manglares en el Caribe presentan una variabilidad determinada por la interacción de las condiciones oceanográficas, climáticas, geomorfológicas y edáficas, las cuales determinan a su vez factores como el nivel y duración de la inundación, la salinidad y la carga de sedimentos que reciben estos bosques (Krauss et al., 2008). Varios estudios han mostrado que los mayores desarrollos en diámetro y altura de los árboles se presentan en aquellos sitios protegidos del impacto directo del oleaje, como bahías y golfos (Suárez, Urrego, Osorio, \& Ruíz, 2015; Urrego, Polanía, Buitrago, Cuartas, \& Lema, 2009), y en los cuales se registran valores bajos a medios de salinidad del agua intersticial y elevadas concentraciones de nutrientes en los suelos, derivada de la entrada 
permanente de agua dulce proveniente de la precipitación o el desbordamiento de ríos y quebradas (Hogarth, 2007), sin mencionar que es en estos sitios donde se presenta la mayor riqueza de especies arbóreas (Ball, 1998; Urrego et al., 2009).

Lugo y Snedaker (1974) generaron la clasificación fisiográfica de manglares del Caribe considerando la interacción de la posición geomorfológica y la distancia a las fuentes de agua dulce. La clasificación ha sido ampliamente utilizada en esta región, debido precisamente a la amplia variabilidad ambiental de los manglares, y a la inexistencia de límites definidos y discretos entre tipos de bosque (Agudelo et al., 2015; Urrego, Molina, \& Suárez, 2014; Urrego et al., 2009). Sin embargo, tanto la variabilidad ambiental como la inexistencia de límites discretos entre tipos de manglares han llevado a varios autores a cuestionar la existencia de una zonación, concepto ampliamente aceptado en el siglo pasado (Dahdouh-Guebas et al., 2002a) para diferentes manglares en varias partes del mundo (Bunt, 1996; Dahdouh-Guebas et al., 2002b).

Aunque todas las especies vegetales del manglar toleran algún grado de salinidad en el suelo y en el agua intersticial (Cintrón \& Schaeffer-Novelli, 1992), cada una de ellas responde de manera distinta a las variaciones en este parámetro. El grado de tolerancia a la salinidad determina la dominancia de las especies dentro del bosque y por consiguiente, contribuye a la diferenciación entre tipos de manglares. Mientras los bosques dominados por Rhizophora mangle (alrededor de 35 tipos de bosque) pueden enfrentar la fuerza del oleaje y salinidades cercanas a las del agua del mar (alrededor de $35 \%$ ), aquellos dominados por Avicennia germinans pueden desarrollarse bajo salinidades de 60-65 \% y sustratos más arenosos. Por otro lado, Laguncularia racemosa (L.) C. F. Gaertn. tolera salinidades similares a las de $R$. mangle, pero requiere de un dosel más abierto, con mayor disponibilidad de luz para su regeneración y por ello se asocia generalmente con sitios perturbados (Benfield, Guzman, \& Mair, 2005).
Además de la salinidad, la composición florística en las comunidades de manglar está también determinada por otras variables como la luz, el drenaje, la dinámica de los sedimentos y la disponibilidad de nutrientes en el suelo (Krauss et al., 2008). Tanto la estructura como la riqueza de especies se maximiza en condiciones de suelos salobres, donde se dispone de más nutrientes y valores de $\mathrm{pH}$ del suelo es más altos (Urrego et al., 2014). En suelos ácidos con altas concentraciones de $\mathrm{Ca}, \mathrm{Mg}, \mathrm{K}, \mathrm{P}$ y carbono orgánico se ha registrado mayor abundancia de Rhizophora, mientras que la abundancia de Avicennia parece ser mayor en suelos menos ácidos, bien drenados y con altas entradas de sedimentos (Urrego et al., 2014).

De esta manera, la caracterización de los manglares de un sitio en particular requiere, además de la evaluación estructural y florística, la identificación y comparación de los gradientes ambientales entre tipos de manglares a nivel local y regional. Sin embargo, esta comparación se vuelve compleja no solo por la dificultad para clasificar estos bosques, sino también por los patrones de fragmentación y degradación a los que están sometidos, dada la creciente perturbación natural y antrópica, que en muchos casos está llevando a la transformación o incluso desaparición de los manglares a escala global (Giri et al., 2011) y en particular en el Caribe Colombiano (Blanco, Estrada, Ortíz, \& Urrego, 2012).

Globalmente se reconoce el actual incremento en el nivel del mar como la mayor amenaza natural que enfrentan los manglares (Alongi, 2015; IPCC, 2014). No obstante, se plantea que los aportes de sedimentos de origen aluvial, especialmente limos y arcillas, son claves para mantener el sustrato y contrarrestar el nivel del mar en aumento (Krauss et al., 2014; McKee, Cahoon, \& Feller, 2007). En el Caribe Colombiano, el nivel del mar ha aumentado alrededor de $20 \mathrm{~cm}$ en los últimos 50 años (Torres, Gómez, \& Afanador, 2006), lo cual ha ocasionado fuertes procesos erosivos y pérdida de manglar en áreas poco protegidas del oleaje (Rangel \& Anfuso, 2009), pero también se han registrado aumentos importantes de las 
extensiones de manglar alojados en deltas y en las orillas de ríos como el Atrato, el Sinú y el Ranchería (Castaño, Urrego \& Bernal, 2010; Suárez et al., 2015; Urrego, Correa-Metrio, González, Castaño, \& Yokoyama, 2013).

En este mismo sentido, los cambios en la cantidad y estacionalidad de la precipitación en el Caribe se constituyen en otras amenazas para estos bosques, puesto que no todas las especies del manglar pueden soportar extensos períodos de sequía, sin mencionar el subsecuente incremento en la salinidad, como los que se registran asociados a la intensificación de El Niño-Oscilación del Sur (ENOS), los cuales generan disminuciones en la productividad de manglares dominados por $R$. mangle y a largo plazo su reemplazo por bosques dominados por A. germinans (Urrego et al., 2013).

Además de los cambios en la precipitación y el nivel del mar, las perturbaciones antrópicas constituyen una grave amenaza para la supervivencia, no solo por la alta demanda de instalaciones turísticas, sino también por los usos que se les ha dado para ganadería, agricultura o expansión de áreas urbanas. Los cambios en el uso del suelo han generado reducciones significativas de estos bosques a lo largo de la costa Caribe colombiana, y altos niveles de contaminación de suelos y aguas de inundación, por el aporte de aguas servidas producto de las deficiencias en la infraestructura para el saneamiento básico en muchas de las áreas que rodean los manglares (Urrego et al., 2014).

Como alternativa para disminuir los impactos de las perturbaciones antrópicas sobre los bosques de manglar, en la última década se han comenzado iniciativas privadas de conservación en diferentes lugares del Caribe Colombiano. Una de ellas es la Reserva Natural Sanguaré, una figura de conservación creada en 2002 que alberga alrededor de 898 ha entre bosques de tierra firme y manglares, derivados de los procesos de recuperación de las coberturas vegetales. Previo al establecimiento de la reserva, estas áreas fueron utilizadas para la ganadería extensiva; y los manglares resultaron intervenidos por la extracción de madera para diferentes usos. Actualmente, el estado de los manglares de la reserva es desconocido, por lo que el objetivo de esta investigación es identificar las variables naturales y antrópicas que determinan la distribución, estructura y composición florística de estos bosques y comparar sus características con otros manglares del Caribe Colombiano, con el fin de aportar herramientas para desarrollar estrategias de manejo y preservación de estos ecosistemas.

\section{MATERIALES Y MÉTODOS}

Área de estudio: Esta investigación se desarrolló en los bosques de manglar de la

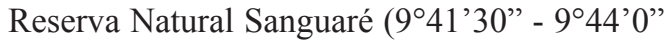

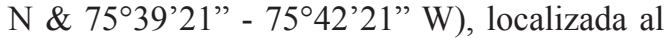
norte del Golfo de Morrosquillo en el municipio de San Onofre, Sucre (Fig. 1). Su altitud varía entre 0-40 $\mathrm{m}$ y posee una precipitación promedio anual de $1000 \mathrm{~mm}$, una evapotranspiración promedio de $1749 \mathrm{~mm}$ y una temperatura media anual de $27^{\circ} \mathrm{C}$. La zona tiene un régimen pluviométrico bimodal, con dos períodos secos (diciembre-abril y julio-septiembre) y dos húmedos (abril-mayo y octubre-noviembre), y corresponde a la zona de vida bosque seco Tropical (bs-T), según la clasificación de Holdridge (1987). La topografía en general es plana y los suelos están conformados por depósitos pleistocénicos de la formación Magdalena y por sedimentos de origen aluvial. El área está rodeada de praderas marinas, manglares y playas, con algunos caños que drenan lagunas costeras como la ciénaga de La Boquilla. La zona no cuenta con corrientes aluviales y en general, el agua dulce proviene de acuíferos superficiales estacionales.

Diseño del muestreo y trabajo de campo: La selección de las áreas de muestreo se realizó por medio de una clasificación visual de las coberturas de la reserva a partir de las imágenes satelitales y aéreas del paquete de capas World Imagery del basemap de ArcGIS (ESRI et al., 2009), lo que permitió identificar diferentes tipos de bosque y elaborar un mapa de coberturas en escala 1:10.000 utilizando ArcGIS 10.3 (ESRI Inc., 10.3). El paquete Worl Imagery está 


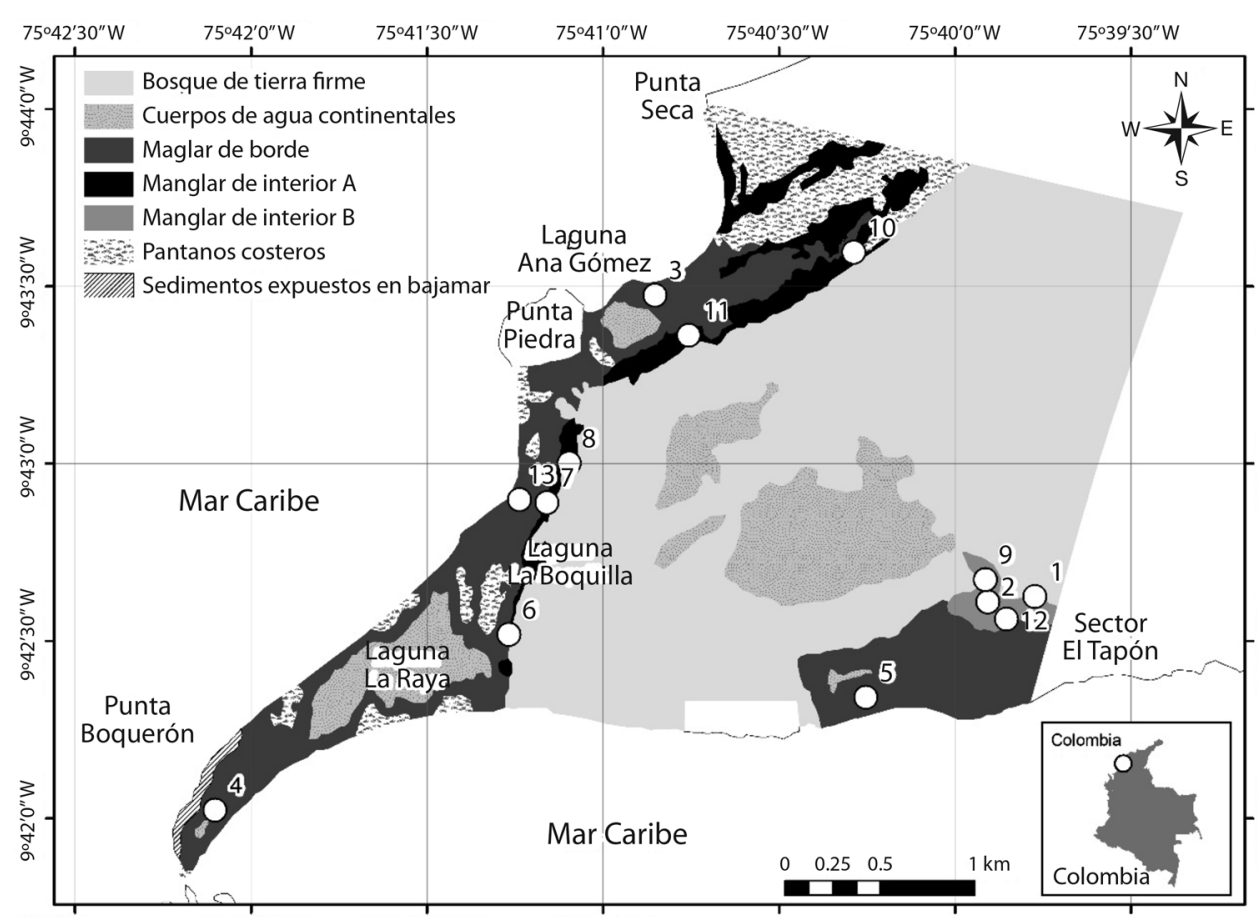

Fig. 1. Área de estudio y distribución de las parcelas de monitoreo - Reserva Natural Sanguaré.

Fig. 1. Study area and distribution of the monitoring plots - Sanguaré Natural Reserve.

compuesto por imágenes satelitales y aéreas de alta resolución $(0.5$ a $15 \mathrm{~m})$ de todo el mundo, las cuales se encuentran entre los 3 y 5 años de vigencia con respecto al periodo analizado. Las coberturas se clasificaron inicialmente con base en la Leyenda Nacional de Coberturas de la Tierra Metodología CORINE Land Cover adaptada para Colombia (IDEAM, 2010) y posteriormente, los bosques de manglar identificados en las imágenes se nombraron como manglares de borde y manglares de interior o cuenca, considerando la clasificación de Lugo y Snedaker (1974). Esta clasificación se utilizó para diseñar el muestreo de campo.

El número de parcelas establecidas por tipo de manglar se calculó a partir de un premuestreo exploratorio (entre cuatro y cinco parcelas establecidas por tipo de manglar dependiendo de la accesibilidad a los sitios), en el cual se determinó la variabilidad del área basal por especie y se estableció un error máximo permisible del $15 \%$.
Se establecieron en total 13 parcelas circulares de $500 \mathrm{~m}^{2}$ (Fig. 1), en las cuales se midieron el diámetro a la altura del pecho (DAP) y la altura total (H) de todos los árboles con DAP superior a $2.5 \mathrm{~cm}$. En el caso de las raíces aéreas ubicadas en un nivel superior a la altura de pecho, el diámetro se midió $30 \mathrm{~cm}$ por encima de la última raíz. Todos los árboles se marcaron e identificaron.

Se midieron en cada parcela, variables ambientales referenciadas en la literatura como determinantes en los bosques de manglar (Krauss et al., 2008; Parida \& Bhavanath, 2010; Reef, Feller, \& Lovelock, 2010; Urrego et al., 2014), incluyendo nivel de inundación o nivel freático, y el $\mathrm{pH}$ y la salinidad el agua intersticial utilizando una Sonda Multiparámetro YSI Professional Pro1030. Para estimar la textura y las concentraciones de nutrientes y materia orgánica (MO), se colectó una muestra de suelo de $1 \mathrm{~kg}$ por parcela una vez durante el estudio, a una profundidad de $25 \mathrm{~cm}$. Las 
muestras se almacenaron en bolsas plásticas marcadas y se enviaron al Laboratorio de Suelos de la Universidad Nacional de Colombia Sede Medellín.

Trabajo de laboratorio: En laboratorio, se realizaron análisis estándar de suelos incluyendo: textura por método de Bouyoucos, $\mathrm{pH}$, capacidad de intercambio catiónico (CIC), contenido de materia orgánica (MO), así como contenidos de macro y micro-nutrientes. Estos análisis se realizaron en el Laboratorio de Suelos de la Universidad Nacional de Colombia utilizando las siguientes normas técnicas colombianas (NTC) y métodos de extracción: NTC_5264 método de extracción suelo/agua (1:1) para pH, NTC_5268 método de saturación con acetato de amonio para CIC, NTC_5403 método de oxidación húmeda para MO \%, NTC_5350 método de Bray II para fósforo (P), NTC 5349 método de acetato de amonio $1 \mathrm{~N}$ y $\mathrm{pH} 7$ para Calcio (Ca), Magnesio $(\mathrm{Mg})$, Sodio $(\mathrm{Na})$ y potasio $(\mathrm{K})$, NTC_5529 método de Olsen modificado para Cobre $(\mathrm{Cu})$, Zinc ( $\mathrm{Zn})$, Hierro (Fe) y Manganeso (Mn), y método Kjeldhal para Nitrógeno $(\mathrm{N})$.

Se construyeron distribuciones diamétricas por especie y por tipo de bosque para caracterizar la estructura de la vegetación. La dominancia de las especies en los tipos de manglar se estimó utilizando el Índice de Valor de Importancia (IVI) propuesto por Curtis y Mcintosh (1951), el cual emplea las abundancias, frecuencias y las dominancias relativas de las especies.

A partir de la información recolectada en campo, se utilizó una prueba de KruskallWallis (Kruskal \& Wallis, 1952) para comparar los parámetros estructurales de la vegetación y las variables ambientales entre tipos de manglar, bajo la hipótesis nula de que las muestras son independientes y provienen de poblaciones idénticas (Johnson, 2012). Para aquellas variables que presentaron diferencias significativas entre tipos de bosques se realizaron pruebas de comparación múltiple usando la prueba de Mann-Whitney (Mann \& Whitney, 1947) acompañada de la corrección de Bonferroni (Bonferroni, 1936).

Adicionalmente, se aplicó un Análisis de Componentes Principales (PCA por sus siglas en inglés), con el fin obtener un número pequeño de combinaciones lineales de las variables ambientales que explicaran la mayor parte de la variabilidad en los datos. Los datos para los cationes de $\mathrm{Na}, \mathrm{K}, \mathrm{Ca}$ y $\mathrm{Mg}$ se incluyeron en el PCA utilizando como variable la relación de salinidad $\mathrm{Cs}=(\mathrm{Na}+\mathrm{K} / \mathrm{Ca}+\mathrm{Mg})$ propuesta por Vegas-Vilarrúbia (2000) para evaluar los patrones espaciales de la vegetación de manglar con base en la salinidad.

Los análisis estadísticos se realizaron con el programa $\mathrm{R}$ versión 3.3.2 (R Core Team, 2016).

\section{RESULTADOS}

Los manglares de borde se ubicaron a lo largo de la costa noroccidental de la reserva entre Punta Piedra y Punta Boquerón, en la zona denominada El Tapón en límites con la Hacienda Los Morros, y en formaciones densas alrededor de las lagunas La Boquilla, La Raya y Ana Gómez; mientras que los de interior se ubicaron detrás de los borde (transición hacia el bosque seco), asociados con playas traseras encharcadas desprovistas de vegetación (Fig. 1). Sin embargo, en el trabajo de campo se identificó un segundo tipo de manglar de interior ubicado en el sector de El Tapón, con mayor desarrollo estructural de los árboles, dominancia de árboles adultos de $A$. germinans y $L$. racemosa, poca regeneración natural y alta influencia aluvial.

Las distribuciones diamétricas totales mostraron forma de "J" invertida truncada para los manglares de borde e interior A, y una distribución asimétrica para los de interior $\mathrm{B}$ (Fig. 2 y Fig. 3). La alta abundancia de árboles de $R$. mangle y de $A$. germinans con $\mathrm{DAP} \leq 20$ $\mathrm{cm}$, y la escasez de árboles grandes (DAP $>20$ $\mathrm{cm}$ ) de estas especies en cualquier grupo caracterizaron las distribuciones en los bosques de borde y de interior A (Fig. 3). En los manglares de interior B, A. germinans y L. racemosa se 


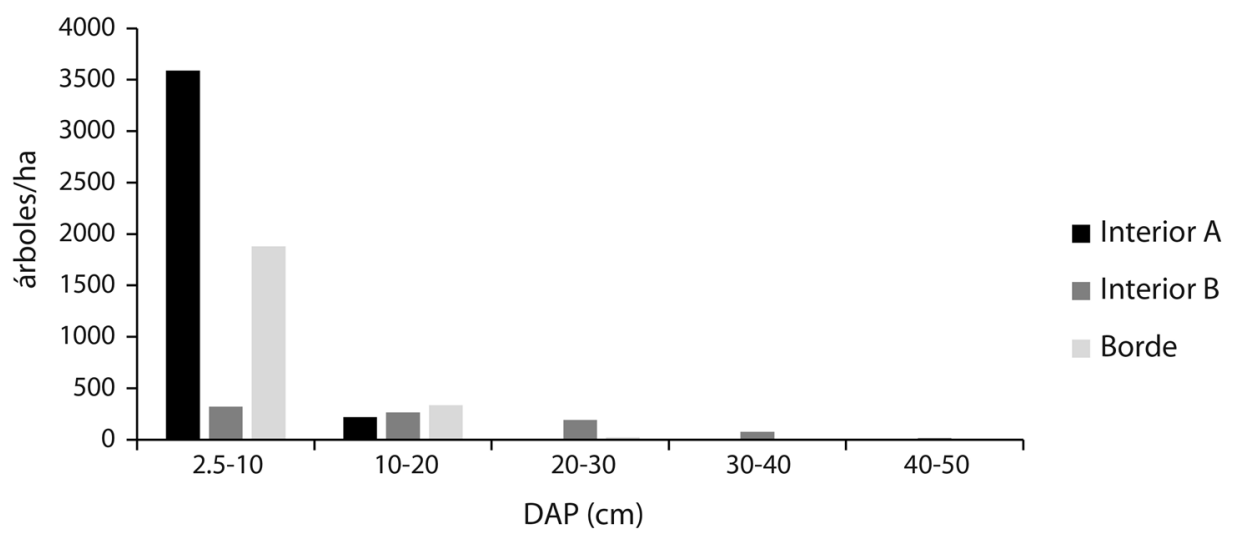

Fig. 2. Distribuciones diamétricas totales por tipo de manglar.

Fig. 2. Total diameter distributions per mangrove type.

TABLA 1

Índice de Valor de Importancia (IVI) de las especies registradas en los tipos de manglar

TABLE 1

Importance Value Index (IVI) of species recorded in mangrove types

\begin{tabular}{lccc}
\multirow{1}{*}{ Especie } & \multicolumn{3}{c}{ Tipo de bosque } \\
& Interior A & Interior B & Borde \\
A. germinans & 264.53 & 214.62 & 13.61 \\
C. erectus & 0.00 & 0.00 & 19.89 \\
L. racemosa & 0.81 & 85.38 & 59.71 \\
R. mangle & 34.66 & 0.00 & 206.79 \\
\hline
\end{tabular}

encontraron también en las categorías diamétricas superiores (DAP $>20 \mathrm{~cm}$ ); sin embargo, $A$. germinans dominó en todas las clases (Fig. 3).

De acuerdo con los resultados del IVI (Tabla 1), $R$. mangle fue la especie más importante en los manglares de borde, mientras que A. germinans lo fue ambos bosques de interior. Aunque $L$. racemosa se encontró en todos los tipos de manglar, su representatividad fue mayor en bosques de borde y de interior B. En los manglares de borde de la zona El Tapón, se encontraron algunos árboles dispersos de Conocarpus erectus L. con diámetros variables.

Se encontraron diferencias significativas entre el DAP promedio (Kruskal-Wallis = $10.68, \mathrm{P}<0.001$ ), la altura promedio (Kruskal-Wallis $=10.68, \mathrm{P}<0.001)$, el área basal (Kruskal-Wallis $=7.83, \mathrm{P}<0.001)$ y el número de árboles por hectárea (Kruskal-Wallis $=9.17$, $\mathrm{P}<0.001)$ entre los 3 tipos de manglar. Los manglares de borde y de interior A presentaron, en general, menor desarrollo estructural, comparados con los de interior B, en los cuales se encontraron valores promedio mayores de DAP, altura y área basal, y menor densidad de árboles. Por otro lado, los bosques de interior A presentaron mayor densidad de individuos, alcanzando valores $3000-4600$ árboles/ha y no tuvieron diferencias significativas en el área basal con respecto a los de borde (Tabla 2).

Se encontraron diferencias significativas entre las variables ambientales de los tres tipos de manglar (Tabla 3). Los manglares de borde se caracterizaron por altas concentraciones $\mathrm{Ca}, \mathrm{P}, \mathrm{Mn}$ y mayores contenidos de materia orgánica; mientras que en los de interior A se encontraron altos niveles de salinidad (82.87 $\pm 9.25 \%)$ y $\mathrm{pH}$ del agua intersticial $(7.19 \pm$ $0.36)$, y mayor proporción de arenas en el suelo $(65.20 \pm 20.28 \%)$. Los manglares de interior $\mathrm{B}$, se caracterizaron por mayor desarrollo de la vegetación en términos de área basal y mayores contenidos de $\mathrm{Cu}, \mathrm{Fe}$ y arcilla en los suelos con respecto a los demás tipos de bosque. La salinidad en este último fue más baja (44.92 \pm $8.96 \%$ ) que en borde e interior A y en algunas parcelas se encontraron trazas de $\mathrm{Al}$ (Tabla 3).

Las comparaciones entre las variables ambientales mostraron diferencias significativas de los bosques borde y de interior A con 

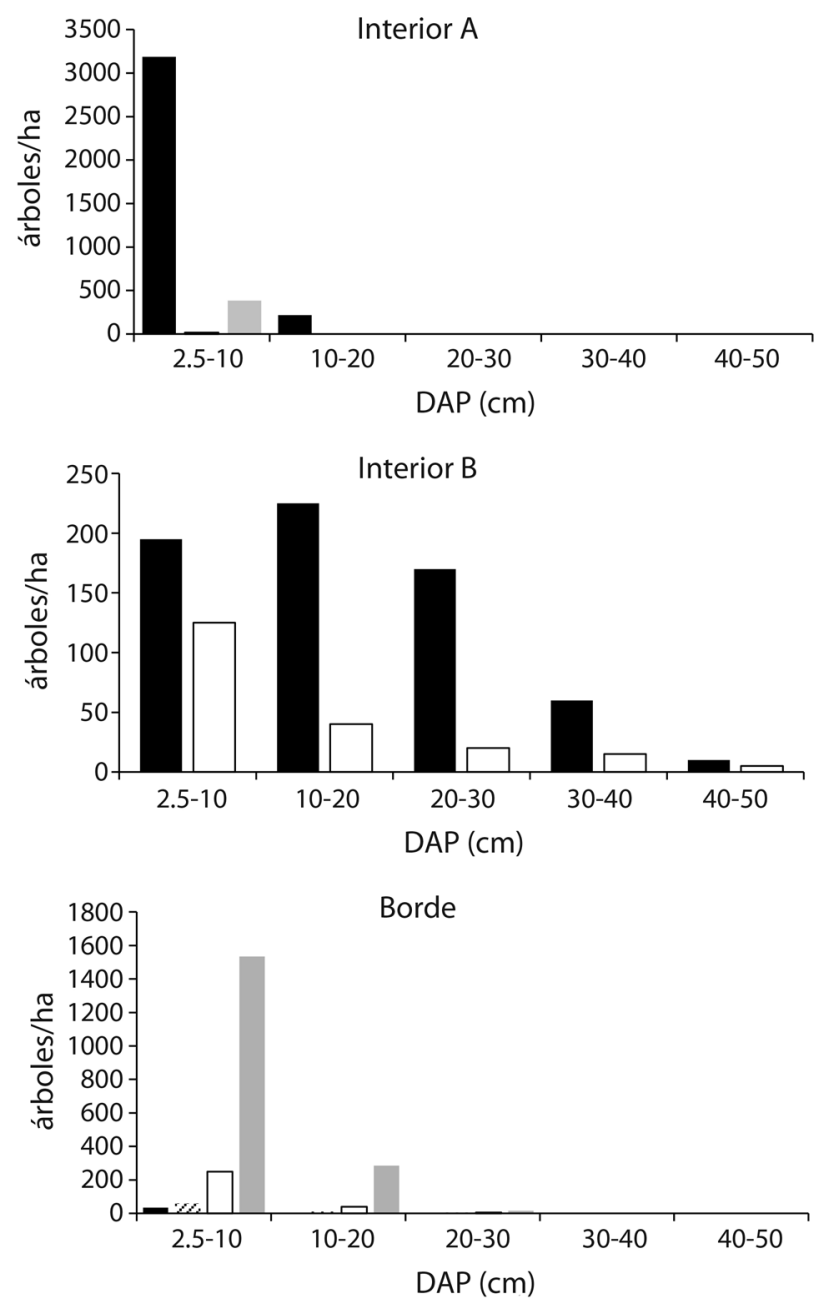

- $\mathrm{Ag} \rightleftharpoons \mathrm{Ce} \square \mathrm{Lr} \square \mathrm{Rm}$

Fig. 3. Distribuciones diamétricas por especie y tipo de manglar.

Fig. 3. Diametric distributions by species and type of mangrove.

respecto a los de interior $\mathrm{B}$, especialmente en la salinidad, el $\mathrm{pH}$ del agua intersticial y los contenidos de $\mathrm{Cu}$ y $\mathrm{Fe}$ en el suelo (Tabla 3), siendo mayores en este último. Las diferencias entre los manglares de borde y de interior (A y B) se relacionaron principalmente con los contenidos de Ca. De igual forma, la salinidad y el $\mathrm{pH}$ del agua intersticial fueron significativamente mayores en borde e interior A los cuales por su ubicación, tienen mayor influencia del agua del mar.
Con respecto al PCA, el primer y segundo eje canónico con auto-valores de 6.48 y 3.65 , respectivamente, explicaron en conjunto el $63.34 \%$ de la variabilidad en los datos (Tabla 4). De manera consistente con los resultados de la prueba de Kruskall-Wallis y el IVI, se observó una separación evidente de los tres tipos de manglar encontrados dentro de la reserva (Fig. 4).

En el componente 1, la salinidad (0.87), el $\mathrm{pH}$ del agua intersticial (0.92), el área basal de 
TABLA 2

Parámetros estructurales de la vegetación de los manglares de la Reserva Natural Sanguaré (media \pm desviación estándar)

TABLE 2

Structural parameters of the vegetation of the mangroves of the Sanguaré Natural Reserve (mean \pm standard deviation)

\begin{tabular}{lccccc} 
& \multicolumn{2}{c}{ Valor promedio } & Área basal $\left(\mathrm{m}^{2} / \mathrm{ha}\right)$ & $\mathrm{Dq}(\mathrm{cm})$ & Densidad (árboles/ha) \\
Tipo de bosque & $\mathrm{DAP}(\mathrm{cm})$ & $\mathrm{Ht}(\mathrm{m})$ & $10.42 \mathrm{a}$ & $5.90 \mathrm{a}$ & $3808 \mathrm{a}$ \\
Interior A & $5.39 \pm 2.41 \mathrm{a}$ & $5.45 \pm 1.75 \mathrm{a}$ & $24.42 \mathrm{~b}$ & $18.96 \mathrm{~b}$ & $865 \mathrm{~b}$ \\
Interior B & $15.67 \pm 10.70 \mathrm{~b}$ & $11.76 \pm 5.88 \mathrm{~b}$ & $10.81 \mathrm{a}$ & $7.84 \mathrm{c}$ & $2240 \mathrm{ab}$ \\
Borde & $7.01 \pm 3.51 \mathrm{a}$ & $7.02 \pm 2.29 \mathrm{a}$ & & \\
\hline
\end{tabular}

DAP: Diámetro a la Altura de Pecho; Ht: Altura total; Dq: Diámetro cuadrático. Las diferencias significativas entre los tres sitios de estudio identificadas con la prueba de Mann-Whitney se indican con las letras a, b y c en cada columna. DAP: Diameter at Breast Height; Ht, Total height; Dq, Quadratic diameter. Significant differences between the three study sites identified with the Mann-Whitney test are indicated by the letters $a, b$, and $\mathrm{c}$ in each column.

TABLA 3

Parámetros ambientales de los manglares de la Reserva Natural Sanguaré (media \pm desviación estándar)

TABLE 3

Environmental parameters of mangrove types of the Sanguaré Natural Reserve (mean \pm standard deviation)

\begin{tabular}{|c|c|c|c|c|}
\hline \multirow{2}{*}{ Parámetro } & \multirow{2}{*}{ Interior $\mathrm{A}(\mathrm{n}=5)$} & \multicolumn{3}{|c|}{ Tipo de bosque } \\
\hline & & Interior $B(n=4)$ & Borde $(n=4)$ & \\
\hline $\mathrm{pH}$ & suelo & $6.56 \pm 0.36 \mathrm{a}$ & $5.78 \pm 0.88 \mathrm{a}$ & $6.68 \pm 0.71 \mathrm{a}$ \\
\hline $\mathrm{pH}$ & agua intersticial & $7.19 \pm 0.36 \mathrm{a}$ & $5.76 \pm 0.26 \mathrm{~b}$ & $6.97 \pm 0.26 \mathrm{a}$ \\
\hline Salinidad $^{1}$ & agua intersticial & $82.87 \pm 9.25 \mathrm{a}$ & $44.92 \pm 8,96 \mathrm{~b}$ & $78.64 \pm 2.88 \mathrm{a}$ \\
\hline Arena $^{2}$ & & $65.20 \pm 20.28 \mathrm{a}$ & $36.50 \pm 24.68 \mathrm{a}$ & $60.00 \pm 14.24 \mathrm{a}$ \\
\hline Limo $^{2}$ & & $27.20 \pm 15.91 \mathrm{a}$ & $32.50 \pm 8.70 \mathrm{a}$ & $27.00 \pm 10.65 \mathrm{a}$ \\
\hline Arcilla $^{2}$ & & $7.60 \pm 4.98 \mathrm{a}$ & $31.00 \pm 17.17 \mathrm{a}$ & $13.00 \pm 7.39 \mathrm{a}$ \\
\hline Materia orgánica² $^{2}$ & & $4.74 \pm 3.49 \mathrm{a}$ & $4.20 \pm 2.36 \mathrm{a}$ & $24.20 \pm 14.48 \mathrm{a}$ \\
\hline $\mathrm{Al}^{3}$ & & - & $0.08 \pm 0.10$ & - \\
\hline $\mathrm{Ca}^{3}$ & & $4.32 \pm 2.59 \mathrm{a}$ & $3.78 \pm 1.67 \mathrm{a}$ & $24.98 \pm 6.78 b$ \\
\hline $\mathrm{Mg}^{3}$ & & $22.72 \pm 14.16 \mathrm{a}$ & $12.18 \pm 7.02 \mathrm{a}$ & $60.98 \pm 36.97 \mathrm{~b}$ \\
\hline $\mathrm{K}^{3}$ & & $2.94 \pm 2.52 \mathrm{ab}$ & $1.17 \pm 0.90 \mathrm{a}$ & $5.63 \pm 3.47 \mathrm{~b}$ \\
\hline $\mathrm{Na}^{3}$ & & $72.36 \pm 50.04 \mathrm{ab}$ & $28.35 \pm 18.38 \mathrm{a}$ & $179.15 \pm 122.61 \mathrm{~b}$ \\
\hline $\mathrm{P}^{4}$ & & $6.80 \pm 3.35 \mathrm{a}$ & $4.75 \pm 2.22 \mathrm{a}$ & $8.00 \pm 6.32 \mathrm{a}$ \\
\hline $\mathrm{Fe}^{4}$ & & $27.00 \pm 4.64 \mathrm{a}$ & $168.50 \pm 87.65 b$ & $13.25 \pm 11.00 \mathrm{a}$ \\
\hline $\mathrm{Mn}^{4}$ & & $2.00 \pm 1.00 \mathrm{a}$ & $5.25 \pm 1.50 \mathrm{a}$ & $20.50 \pm 31.82 \mathrm{a}$ \\
\hline $\mathrm{Cu}^{4}$ & & $1.60 \pm 0.55 \mathrm{a}$ & $4.00 \pm 1.15 \mathrm{~b}$ & $1.00 \pm 0.00 \mathrm{a}$ \\
\hline $\mathrm{Zn}^{4}$ & & $1.80 \pm 0.84 \mathrm{a}$ & $2.75 \pm 2.22 \mathrm{a}$ & $1.75 \pm 0.96 \mathrm{a}$ \\
\hline $\mathrm{N}^{2}$ & & $0.12 \pm 0.12 \mathrm{a}$ & $0.19 \pm 0.08 \mathrm{a}$ & $0.83 \pm 0.55 b$ \\
\hline
\end{tabular}

$1 \% \quad 2 \% \quad{ }^{3} \mathrm{cmolc} / \mathrm{Kg} \quad{ }^{4} \mathrm{mg} / \mathrm{Kg}$.

Las diferencias significativas entre los tres sitios de estudio identificadas con la prueba de Mann-Whitney se indican con las letras a, b y c en cada fila. Significant differences between the three study sites identified with the Mann-Whitney test are indicated by the letters $\mathrm{a}, \mathrm{b}$, and $\mathrm{c}$ in each row. 


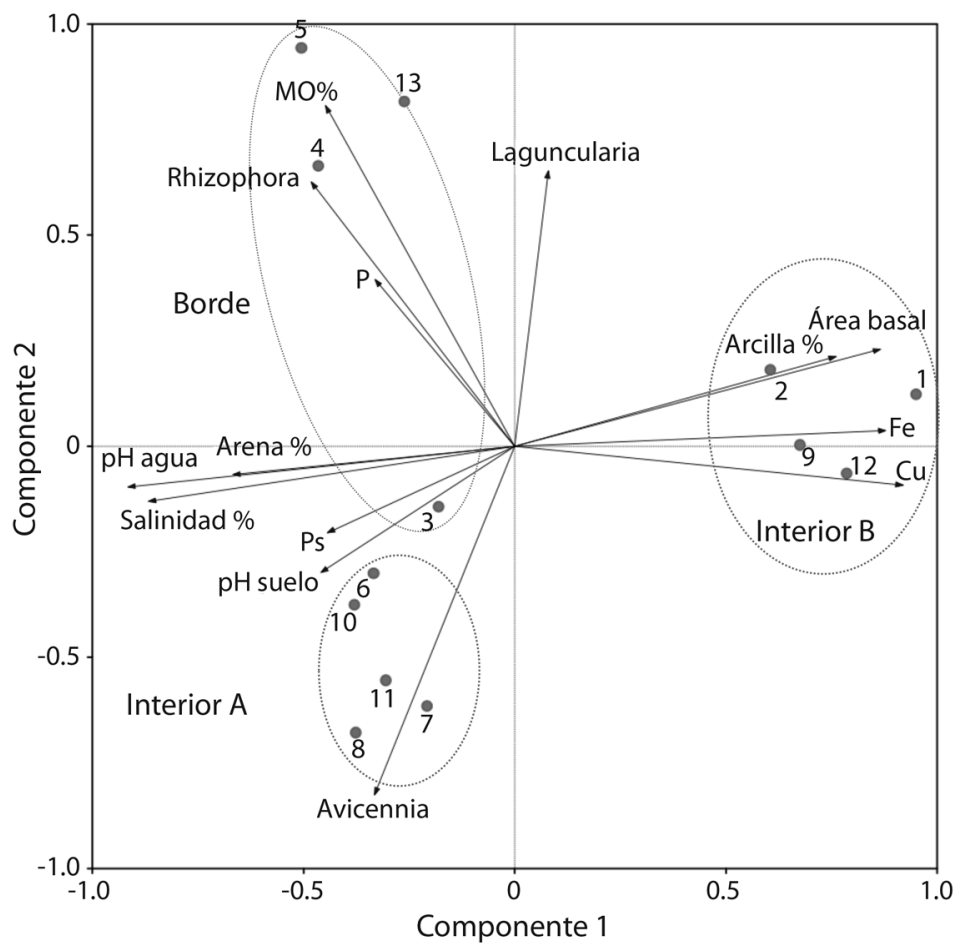

Fig. 4. Análisis de Componentes Principales de 13 parcelas permanentes con 3 especies de mangle y 14 variables físicas en la Reserva Natural Sanguaré.

Fig. 4. Principal Component Analysis (PCA) of 13 permanent plots with 3 mangrove species and 14 physical variables in the Sanguaré Natural Reserve.

TABLA 4

Desviación estándar y varianza explicada del Análisis de Componentes Principales

TABLE 4

Standard deviation and explained variance of Principal Component Analysis

\begin{tabular}{lcccc}
\multicolumn{1}{c}{ Estadístico } & \multicolumn{5}{c}{ Componentes } \\
\hline Desviación estándar & 1 & 2 & 3 & 1.68 \\
Varianza explicada (\%) & 6.11 & 2.97 & 11.20 & 1.44 \\
Varianza acumulada (\%) & 40.75 & 19.82 & 71.78 & 9.61 \\
\hline
\end{tabular}

los árboles (-0.88) y los contenidos de $\mathrm{Fe} \mathrm{y} \mathrm{Cu}$ (0.88 y 0.91 , respectivamente), presentaron los valores más altos de correlación y permitieron la separación de los manglares de borde y de interior $\mathrm{A}$, de los de interior B. Estos últimos presentaron los mayores valores de $\mathrm{Fe}, \mathrm{Cu}$ y arcilla en los suelos, y la mayor área basal en la vegetación.
A lo largo del componente 2, se separaron los manglares de borde y de interior A. Mientras los bosques de borde se asociaron a mayores valores en los porcentajes de materia orgánica, concentraciones de $\mathrm{P}$ y $\mathrm{Mn}$ en los suelos, y a la dominancia de $R$. mangle y L. racemosa; los de interior A se asociaron a mayores valores de $\mathrm{pH}$ del suelo y del agua 
intersticial, mayor salinidad y mayor porcentaje de arena en los suelos, así como a la dominancia de $A$. germinans.

\section{DISCUSIÓN}

Los manglares de la Reserva Natural Sanguaré se encuentran dentro de un rango de variación estructural y florística similar al de otros manglares en el Caribe. Sin embargo, las diferencias entre los tipos de manglar encontrados y de éstos con otros del Caribe Colombiano (Ulloa-Delgado, Gil-Torres, Pino-Rengifo, \& Rodríguez-Cruz, 1998) y de Centroamérica (Pool, Snedaker, \& Lugo, 1977), reflejan las presiones ejercidas por las condiciones ambientales y las perturbaciones naturales y antrópicas.

En términos estructurales, los manglares de borde y de interior A en la reserva, presentaron valores promedio de diámetros y alturas relativamente bajos, comparables a los reportados en manglares en regeneración o muy intervenidos, como los que se encuentran en algunas zonas del Golfo de Urabá (Urrego et al., 2014). En estos últimos, la extracción permanente de los árboles más grandes de $R$. mangle ha generado en algunas zonas la dominancia de árboles de tallas pequeñas, lo cual coincide con las distribuciones diamétricas en forma de jota invertida truncada de ambos tipos de bosque (borde e interior A), y con registros de otros manglares con perturbación antrópica (LópezHoffman, Monroe, Narváez, Martínez-Ramos, \& Ackerly, 2006). No obstante, las diferencias en composición florística y las características abióticas de ambos manglares, pueden explicar el comportamiento de estas distribuciones.

En los manglares de borde ubicados en los sectores de La Punta, se registró una colonización de $R$. mangle, resultado del cierre de las lagunas La Boquilla y La Raya, lo cual disminuye la energía del oleaje y facilita el depósito o acumulación de sedimentos en los alrededores o en las áreas circunvecinas. A nivel de estos sedimentos se presenta la colonización de manglar joven tal como se ha presentado en el proceso de formación de otras lagunas costeras en el Caribe (Urrego et al., 2013). Este proceso está acompañado por el desplazamiento de la línea de costa tierra-adentro derivado del incremento en el nivel del mar que se viene registrando desde principios de los 90's (Torres et al., 2006).

La regeneración y colonización de $R$. mangle en manglares de borde, se ha registrado en el Caribe colombiano en La Caimanera, La Ensenada de Río Negro en el Golfo de Urabá (Suárez et al., 2015; Urrego et al., 2014) y la bahía de Cispatá (Agudelo et al., 2015), lo cual ha llevado a la expansión de estos manglares en las últimas décadas, especialmente en sitios con aportes aluviales, como en los deltas y las orillas de ríos y quebradas, y en sitios tierradentro donde hay espacio disponible para su expansión. Sus características estructurales están dentro del rango de manglares en regeneración como los que se encuentran en el archipiélago de las perlas en Panamá (McGowan et al., 2010).

En los manglares de borde de la reserva, se presentan alta concentración de $\mathrm{Ca}$ y $\mathrm{Mg}$ en los suelos lo que indica la predominancia de los aportes marinos sobre los aluviales como en algunos manglares del Golfo de Urabá (Urrego et al., 2014). En estos bosques también se presentaron los mayores porcentajes de $\mathrm{P}$ y materia orgánica como resultado de la lenta descomposición de la hojarasca proveniente de la caída de material vegetal de los árboles de $R$. mangle (Kathiresan \& Bingham, 2001; Lima \& Colpo, 2014).

No obstante, la extracción de madera previa al establecimiento de la reserva puede dar cuenta de la ausencia de árboles de diámetros mayores en los manglares de borde de mayor edad tal como se registró en el Golfo de Urabá y en la bahía de Cispatá (Agudelo et al., 2015; Urrego et al., 2014). Tal extracción se presenta comúnmente en manglares cercanos a los centros poblados y a las corrientes de agua que facilitan su aprovechamiento y por ello es común la relación directa entre el aumento del área basal o el diámetro promedio con la distancia a tales poblados (Suárez et al., 2015). Esta perturbación también explica la presencia de L. racemosa; especie más frecuente en 
manglares intervenidos cercanos a los caseríos y centros poblados (Benfield et al., 2005).

Los manglares de interior $\mathrm{A}$, localizados detrás del manglar de borde, hacia tierra adentro, se asemejan a los llamados manglares de playa trasera descritos por (Von Prahl, Cantera, \& Contreras, 1990). En ellos, las condiciones de suelos arenosos con alta salinidad (82.87 $\pm 9.25 \%$ ), restringen el desarrollo estructural del bosque, lo cual explica los bajos valores promedio de altura y diámetro de los árboles. La elevada salinidad también limita el establecimiento de especies diferentes a $A$. germinans, y por eso es ésta la especie dominante, tal como se registra en manglares de interior del Golfo de Urabá, la bahía de Cispatá, la Ciénaga Grande de Santa Marta y La Guajira (Agudelo et al., 2015; Ulloa-Delgado et al., 1998; Urrego et al., 2014; Vásquez, 2000).

Aunque algunos autores atribuyen la alta abundancia de juveniles, la baja área basal total y el dosel muy abierto a la perturbación antrópica (Walters, 2005b), este comportamiento también es común en manglares de interior en zonas áridas donde la baja precipitación y nivel freático, y la alta evaporación, posibilitan la dominancia de $A$. germinans de menor porte (Méndez-Linares, López-Portillo, Hernández-Santana, \& Ortíz-Pérez, 2007). Las distribuciones diamétricas evidencian el estado sucesional temprano de los manglares de borde y de interior A dentro de la reserva, y muestran que los rodales de interior B son más antiguos.

El establecimiento del manglar de interior detrás del manglar de borde se asocia a factores conocidos y también presentes en la reserva, como el reemplazo del suelo orgánico, con mayor inundación y exposición a la influencia del oleaje donde domina $R$. mangle, por suelos arenosos, menos inundados y más salinos donde domina $A$. germinans (Rajkaran $\&$ Adams, 2012) siguiendo un patrón clásico de zonación encontrado en otros manglares del mundo (Ellison, Mukherjee, \& Karim, 2000).

A diferencia de los manglares de borde e interior $\mathrm{A}$, los bosques de interior $\mathrm{B}$ presentaron un mayor desarrollo estructural evidenciado por los altos valores de diámetro y altura promedio. De esta manera, los manglares de interior B corresponden con bosques maduros asociados a una menor salinidad y mayores contenidos de arcilla en los suelos (Fig. 4). No obstante, la escasa representatividad de árboles jóvenes, y la presencia de L. racemosa, reflejan una alta intervención antrópica (Walters, 2005a) que probablemente se realizó antes del establecimiento de la reserva, dada la ausencia de extensiones amplias del helecho Acrostichum aureum L., especie heliófila, indicadora de apertura del dosel.

La localización de los manglares de interior $\mathrm{B}$, en cercanías a varios establecimientos turísticos, puede explicar las mayores concentraciones de $\mathrm{Fe}$ y $\mathrm{Cu}$ como resultado de la contaminación de los suelos y de las aguas de inundación, por la recepción de aguas servidas. A pesar de la amplia variación espacial que pueden tener las concentraciones de varios elementos en los suelos (Otero, Ferreira, VidalTorrado, \& Macías, 2006), las altas concentraciones de $\mathrm{Cu}$ y otros metales pesados se han asociado a manglares con alta perturbación antrópica (Lu \& Chen, 1977; Singh, Ranjan, Chauhan, \& Ramanathan, 2010) y a un origen de la contaminación similar al registrado en Sanguaré.

La deficiencia en la red de alcantarillado de las viviendas que bordean los manglares de interior B, puede estar afectando el desarrollo de la vegetación y especialmente de plántulas y juveniles. La contaminación de las aguas que inundan los manglares por aporte de aguas servidas no solo puede aumentar la concentración de metales pesados en los suelos (especialmente $\mathrm{Fe} \mathrm{y} \mathrm{Cu}$ ), sino también alterar los ciclos biogeoquímicos de otros elementos como el azufre, como se ha reportado en otros manglares del mundo (Prasad \& Ramanathan, 2008). Especialmente en países en desarrollo, las comunidades humanas asentadas cerca a los bosques de manglar poseen un alto grado de necesidades básicas insatisfechas y altas deficiencias en la infraestructura de saneamiento básico como se observa en Sanguaré, en el Golfo de Urabá y en La Guajira, en Colombia (Urrego et al., 2014). 
Los manglares de interior B en Sanguaré, presentaron mayor área basal comparados con los manglares de borde, tal como se registró en la bahía de Cispatá (Agudelo et al., 2015). En el Caribe Colombiano, los valores del área basal en manglares de interior son generalmente mayores que en los manglares de borde en zonas como La Guajira, la Ciénaga Grande de Santa Marta, la bahía de Cispatá y el Golfo de Urabá, en Colombia (Agudelo et al., 2015; Ulloa-Delgado et al., 1998; Urrego et al., 2014). En todos los casos anteriores se atribuye la dominancia de $A$. germinans, bajo salinidades intermedias (40-50\%), a la intensa explotación de $R$. mangle para la construcción de viviendas (Suárez et al., 2015).

A pesar del establecimiento del área de reserva en Sanguaré, en la actualidad se presenta tala selectiva de especies de R.mangle dada la fácil accesibilidad de los pobladores de la zona a los manglares, y por el alto valor de su madera para la construcción y la producción de carbón y leña. Los valores de área basal en manglares de interior B son también mayores que los registrados en otros bosques de interior (cuenca) como los de las islas de San Andrés (Colombia), México, Costa Rica y Puerto Rico (Pool et al., 1977; Urrego et al., 2009).

A manera de conclusión, la composición florística y la estructura de los manglares de la Reserva Natural Sanguaré presentan una estrecha relación con características ambientales particulares como la salinidad, las condiciones edáficas, el pH y la acumulación de materia orgánica; sin embargo, estos manglares, al igual que otros bosques del Caribe y del mundo, han sido afectados por la contaminación y la fuerte perturbación antrópica que los ha reducido significativamente, y también ha dado lugar a la generación de manglares con condiciones físicas altamente restrictivas para el desarrollo de la vegetación (Agudelo et al., 2015; Urrego et al., 2014), las cuales deberán ser tomadas en cuenta en la articulación de los Planes de Manejo de las figuras de conservación como la Reserva Natural Sanguaré.
Declaración de ética: los autores declaran que todos están de acuerdo con esta publicación y que han hecho aportes que justifican su autoría; que no hay conflicto de interés de ningún tipo; y que han cumplido con todos los requisitos y procedimientos éticos y legales pertinentes. Todas las fuentes de financiamiento se detallan plena y claramente en la sección de agradecimientos. El respectivo documento legal firmado se encuentra en los archivos de la revista.

\section{AGRADECIMIENTOS}

Se agradece a la Universidad Nacional de Colombia Sede Medellín por el financiamiento otorgado a través del Programa Nacional de Proyectos para el Fortalecimiento de la Investigación, la Creación y la Innovación en Posgrados de la Universidad Nacional de Colombia 2013 - 2015. A los directivos y empleados de la Reserva Natural Sanguaré por la colaboración y logística en la fase de campo. A Melisa Maya Mesa por su colaboración en el levantamiento de las parcelas y la toma de datos en campo.

\section{RESUMEN}

Los manglares presentan diferentes grados de interacción entre las condiciones oceanográficas, climáticas, geomorfológicas y edáficas. Estas interacciones tienen repercusiones fuertes sobre la condiciones locales de salinidad y disponibilidad de agua y nutrientes a nivel local, y sobre la estructura y composición de los manglares. Con base en el análisis de fotografías aéreas, muestreo en campo y análisis de componentes principales, se analizaron la composición florística y la estructura de los manglares presentes en la Reserva Natural Sanguaré. Se identificaron tres tipos de bosques según la clasificación fisiográfica de manglares. Un manglar de borde dominado por Rhizophora mangle L. en estado juvenil, con bajo desarrollo estructural, y dos tipos de manglar de interior o cuenca dominados por Avicennia germinans (L.) L. El primero de ellos, localizado detrás del manglar de borde, presentó alta salinidad en el suelo y en el agua intersticial, predominancia de sedimentos arenosos, y bajos valores de altura y diámetro a la altura de pecho (DAP) promedio de los árboles. El segundo, con un bajo número de árboles adultos en todas 
las categorías de diámetro, mayor área basal, bajo potencial de regeneración natural, y altas concentraciones de $\mathrm{Fe} \mathrm{y} \mathrm{Cu}$ en el suelo, probablemente asociado a la contaminación por vertimientos domésticos. La ubicación del primer manglar de interior detrás del manglar de borde revela la zonación típica descrita para muchos manglares del mundo. La composición florística, la estructura y la distribución de los manglares en la Reserva Sanguaré son el resultado de la interacción de variables ambientales locales como la salinidad y la textura del suelo, además de la intervención antrópica previa al establecimiento de la reserva y la actual presencia humana muy cerca de los manglares.

Palabras clave: manglar; regeneración; contaminación; variabilidad natural; conservación.

\section{REFERENCIAS}

Agudelo, C. M., Bolívar, J., Polanía, J., Urrego, L. E., Yépes, A., \& Sierra, A. (2015). Estructura y composición florística de los manglares de la bahía de Cispatá, Caribe Colombiano. Revista de Biología Tropical, 63, 1137-1147.

Alongi, D. M. (2015). The impact of Climate Change on mangrove forests. Current Climate Changes Report, 1, 30-39. https://doi.org/10.1007/s40641-015-0002-x

Ball, M. C. (1998). Mangrove species richness in relation to salinity and waterlogging: a case study along Adelaide River floodplain, northern Australia. Global Ecology and Biogeography, 7, 73-82.

Benfield, S. L., Guzman, H. M., \& Mair, J. M. (2005). Temporal mangrove dynamics in relation to coastal development in Pacific Panama. Journal of Environmental Management, 76, 263-276.

Blanco, J. F., Estrada, E. A., Ortíz, L. F., \& Urrego, L. E. (2012). Ecosystem-wide impacts of deforestation in mangroves: the Urabá Gulf (Colombian Caribbean) case study. https://doi.org/10.5402/2012/958709

Bonferroni, C. E. (1936). Teoria statistica delle classi e calcolo delle probabilità. Pubblicazioni del R Istituto Superiore di Scienze Economiche e Commerciali di Firenze, 8, 3-62.

Bunt, J. S. (1996). Mangrove Zonation: an examination of data from seventeen riverine estuaries in tropical Australia. Annals of Botany, 78, 333-341.

Castaño, A. R., Urrego, L. E., \& Bernal, G. (2010). Dinámica del manglar en el complejo lagunar de Cispatá (Caribe Colombiano) en los últimos 900 años. Revista de Biología Tropical, 58, 1347-1366.

Cintrón, G., \& Schaeffer-Novelli, Y. (1992). Ecology and management of new world mangroves. In U. Seeliger (Ed.), Coastal plant communities of Latin America (pp. 233-258). San Diego, USA: Academic Press.
Curtis, J. T., \& Mcintosh, R. P. (1951). An upland forest continuum in the prairie-forest border region of Wisconsin. Ecology, 32, 476-496.

Dahdouh-Guebas, F., Kairo, J., Jayatissa, L. P., Cannicci, S., \& Koedam, N. (2002a). An ordination study to view vegetation structure dynamics in disturbed and undisturbed mangrove forests in Kenya and Sri Lanka. Plant Ecology, 161, 123-135.

Dahdouh-Guebas, F., Verneirt, M., Cannicci, S., Kairo, J. G., Tack, J. F., \& Koedam, N. (2002b). An exploratory study on grapsid crab zonation in Kenyan mangroves. Wetlands Ecology and Management, 10, 179-187.

Ellison, A. M., Mukherjee, B. B., \& Karim, A. (2000). Testing patterns of zonation in mangroves: scale dependence and environmental correlates in the Sundarbans of Bangladesh. Journal of Ecology, 88, 813-824.

Esri, DigitalGlobe, GeoEye, Earthstar Geographics, CNES/ Airbus DS, USDA, USGS, AeroGRID, IGN \& the GIS User Community. (2009). World Imagery Map Service. https://services.arcgisonline.com/ArcGIS/ rest/services/World_Imagery/MapServer.

ESRI Inc., R., CA. (10.3). Corporate headquarters. ESRI. 380 New York Street Redlands, 92373-8100. ArcGIS, 10.3 .

Giri, C., Ochieng, E., Tieszen, L. L., Zhu, Z., Singh, A., Loveland, T., Masek, J. \& Duke, N. (2011). Status and distribution of mangrove forests of the World using Herat observation satellite data. Global Ecology and Biogeography, 20, 154-159.

Hogarth, P. (2007). The biology of mangroves and seagrasses (2nd Ed.). New York, USA: Oxford University Press.

Holdridge, L. (1987). Ecología basada en zonas de vida. San José, Costa Rica: Instituto Interamericano de Cooperación para la Agricultura.

IDEAM. (2010). Leyenda Nacional de Coberturas de la Tierra. Metodología CORINE Land cover adaptada para Colombia Escala 1:100.000. Bogotá, D. C.: Instituto de Hidrología, Meteorología y Estudios Ambientales.

IPCC. (2014). Climate Change 2014: contribution of working groups I, II and III to the fifth assessment report of the Intergovernmental Panel on Climate Change. Geneva, Switzerland. Retrieved from https://www. ipcc.ch/pdf/assessment-report/ar5/syr/SYR_AR5 FINAL_full_wcover.pdf

Johnson, R. A. (2012). Probabilidad y estadística para ingenieros. México, México: Pearson Education. 
Kathiresan, K., \& Bingham, B. L. (2001). Biology of mangroves and mangrove ecosystems. Advances in Marine Biology, 40, 81-251.

Krauss, K. W., Lovelock, C. E., Mckee, K. L., López-Hoffman, L., Ewe, S. M. L., \& Sousa, W. P. (2008). Environmental drivers in mangrove establishment and early development: a review. Aquatic Botany, 89, 105127. https://doi.org/10.1016/j.aquabot.2007.12.014

Krauss, K. W., McKee, K. L., Lovelock, C. E., Cahoon, D. R., Saintilan, N., Reef, R., \& Chen, L. (2014). How mangrove forests adjust to rising sea level. New Phytologist, 202, 19-34.

Kruskal, W. H. \& Wallis, A. W. (1952). Use of ranks in onecriterion variance analysis. Journal of the American Statistical Association, 47, 583-621.

Lima, R. G., \& Colpo, K. D. (2014). Leaf-litter decomposition of the mangrove species Avicennia schaueriana, Laguncularia racemosa and Rhizophora mangle. Journal of the Marine Biological Association of the United Kingdom, 94, 233-239.

López-Hoffman, L., Monroe, I. E., Narváez, M., MartínezRamos, M., \& Ackerly, D. D. (2006). Sustainability of mangrove harvesting: how do harvesters' perceptions differ from ecological analysis? Ecology and Society, 11, 18-23.

Lu, C. S. J., \& Chen, K. Y. (1977). Migration of trace metals in interfaces of seawater and polluted surficial sediments. Environmental Science and Technology, 11, 174-182.

Lugo, A. E., \& Snedaker, S. C. (1974). The ecology of mangroves. Annual Review of Ecology and Systematics, 5, 39-64.

Mann, H. B. \& Whitney, D. R. (1947). On a test of whether one of two random variables is stochastically larger than the other. The Annals of Mathematical Statistics, $18,50-60$.

McGowan, T., Cunningham, S., Guzmán, H. M., Mair, J., Guevara, J. M., \& Betts, T. (2010). Mangrove forest composition and structure in Las Perlas Archipelago, Pacific Panama. Revista de Biología Tropical, 58 , 857-869.

McKee, K. L., Cahoon, D. R., \& Feller, I. C. (2007). Caribbean mangroves adjust to rising sea level through biotic controls on change in soil elevation. Global Ecology and Biogeography, 16, 545-556.

Méndez-Linares, A. P., López-Portillo, J., HernándezSantana, J. R., \& Ortíz-Pérez, M. A. (2007). The mangrove communities in the Arroyo Seco deltaic fan, Jalisco, Mexico, and their relationship with geomorphic and physical-geographic zonation. Catena, $70,127-142$
Otero, X. L., Ferreira, T. O., Vidal-Torrado, P., \& Macías, F. (2006). Spatial variation in pore water geochemistry in a mangrove system (Pai Matos Island, CananeiaBrazil). Applied Geochemistry, 21, 2171-2186.

Parida, A. K., \& Bhavanath, J. (2010). Salt tolerance mechanisms in mangroves: a review. Trees, 24, 199. 217. https://doi.org/10.1007/s00468-010-0417-x

Pool, D., Snedaker, S. C., \& Lugo, A. E. (1977). Structure of mangrove forests in Florida, Puerto Rico, Mexico and Costa Rica. Biotropica, 9, 195-212.

Prasad, M. B. K., \& Ramanathan, A. L. (2008). Sedimentary nutrient dynamics in a tropical estuarine mangrove ecosystem. Estuarine, Coastal and Shelf Science, 80, 60-66.

R Core Team (2016). $R$ 3.3.3 for windows: A language and environment for statistical computing. R Foundation for Statistical Computing, Vienna, Austria. http:// www.R-project.org/

Rajkaran, A., \& Adams, J. (2012). The effects of environmental variables on mortality and growth of mangroves at Mngazana Estuary, Eastern Cape, South Africa. Wetlands Ecology and Management, 20, 297-312.

Rangel, N., \& Anfuso, G. (2009). Assessment of coastal vulnerability in La Guajira Peninsula Colombian Caribbean Sea. Journal of Coastal Research, 56, 792-796.

Reef, R., Feller, I. C., \& Lovelock, C. E. (2010). Nutrition of mangroves. Tree Physiology, 30, 1148-1160. https://doi.org/10.1093/treephys/tpq048

Singh, G., Ranjan, R. K., Chauhan, R., \& Ramanathan, A. L. (2010). Dissolved metal distribution in Indian mangrove ecosystem: case studies from East Coast of India. In Management and sustainable development of coastal zone environments. Berlin, Germany: Springer.

Suárez, J. A., Urrego, L. E., Osorio, A., \& Ruíz, H. Y. (2015). Oceanic and climatic drivers of mangrove changes in the Gulf of Urabá, Colombian Caribbean. Latin American Journal of Aquatic Research, 43, 972-985.

Torres, R. R., Gómez, J. C., \& Afanador, F. (2006). Variación del nivel medio del mar en el Caribe Colombiano. Boletín Cientifico CIOH, 24, 64-72.

Ulloa-Delgado, G. A., Gil-Torres, W. O., Pino-Rengifo, J. C., \& Rodríguez-Cruz, H. (1998). Manual sobre técnicas de vivero y restauración de áreas de manglar del Caribe Colombiano. Bogotá, D. C., Colombia: Proyecto PD 171/91 Rev. 2 Fase II (Etapa I) Conservación y Manejo para el Uso Múltiple y el Desarrollo de los Manglares en Colombia, MMA / ACOFORE / OIMT. 
Urrego, L. E., Correa-Metrio, A., González, C., Castaño, A. R., \& Yokoyama, Y. (2013). Contrasting responses of two Caribbean mangroves to sea-level rise in the Guajira Peninsula (Colombian Caribbean). Palaeogeography, Palaeoclimatology, Palaeoecology, 370, 92-102.

Urrego, L. E., Molina, E., \& Suárez, J. A. (2014). Environmental and anthropogenic influences on the distribution, structure, and floristic composition of mangrove forests of the Gulf of Urabá (Colombian Caribbean). Aquatic Botany, 114, 42-49. https://doi.org/10.1016/j. aquabot.2013.12.006

Urrego, L. E., Polanía, J., Buitrago, M. F., Cuartas, L. F., \& Lema, Á. (2009). Distribution of mangroves along environmental gradients on San Andres Island (Colombian Caribbean). Bulletin of Marine Science, $85,27-43$.
Vásquez, J. L. (2000). Estructura de los bosques de mangle del Valle de los Cangrejos y el Riíto, delta del río Ranchería, Caribe Colombiano. Universidad Nacional de Colombia Sede Medellín.

Vegas-Vilarrúbia, T. (2000). Zonation pattern of an isolated mangrove community at Playa Medina, Venezuela. Wetlands Ecology and Management, 8, 9-17.

Von Prahl, H., Cantera, J., \& Contreras, R. (1990). Manglares y Hombres del Pacífico colombiano. Bogotá, D. C., Colombia.

Walters, B. (2005a). Ecological effects of small-scale cutting of Philippine mangrove forests. Forest Ecology and Management, 206, 331-348.

Walters, B. (2005b). Patterns of local wood use and cutting of Philippine mangrove forests. Economic Botany, 59, 66-76. 